\title{
Volume 184, no. 14, 2002, Table of Contents (print version)
}

Table of Contents: The title and authors for the erratum should appear as shown below.

The ner Gene of Photorhabdus: Effects on Primary-Form-

Specific Phenotypes and Outer Membrane Protein

Composition
Keith H. O’Neill, Declan M. Roche, David J. Clarke, and Barbara C. A.

Dowds 